\title{
Isoquinoline-Catalyzed Reaction between 4-Hydroxycoumarin or 4-Hydroxy-6-methylpyran-1-one and Dialkyl Acetylene Dicarboxylates: Synthesis of Coumarin and Pyranopyrane Derivatives
}

\author{
Mohammad Anary-Abbasinejad, ${ }^{*, a}$ Hossein Anaraki-Ardakani, ${ }^{a}$ \\ Mohammad Hossein Mosslemin ${ }^{a}$ and Hamid Reza Khavasi ${ }^{b}$
}

\author{
${ }^{a}$ Department of Chemistry, Islamic Azad University, Yazd Branch, PO Box 89195-155, Yazd, Iran \\ ${ }^{b}$ Department of Chemistry, Shahid Beheshti University, PO Box 1983963113, Tehran, Iran
}

\begin{abstract}
Neste trabalho, relatamos a reação entre dialquil acetilenodicarboxilatos e sistemas enólicos tais como 5,5-dimetil-1,3-ciclohexanodiona, 1,3-ciclohexanodiona, 4-hidroxicumarina ou 4-hidróxi6-metilpiran-1-ona na presença de isoquinolina, a qual leva a novos derivados de cumarina e piranopirano.
\end{abstract}

In this work we report the reaction between dialkyl acetylenedicarboxylates and enolic systems such as 5,5-dimethyl-1,3-cyclohexanedione, 1,3-cyclohexanedione, 4-hydroxycoumarin or 4-hydroxy-6-methylpyran-1-one in the presence of isoquinoline, which leads to new coumarin and pyranopyran derivatives.

Keywords: acetylenedicarboxylic esters, isoquinoline, pyranopyran derivatives, coumarin derivatives

\section{Introduction}

Coumarin is the structural motif of many natural and synthetic compounds that endows them with a wide range of biological activities. Given the development of coumarins as photosensitizers, ${ }^{1}$ anti-HIV agents, ${ }^{2}$ antibiotics, ${ }^{3}$ rodenticides, and oral anticoagulants, ${ }^{4}$ there is continuing interest in the synthesis of these materials.

The rich and fascinating chemistry stems from the addition of nucleophiles to activated acetylene compounds has evoked considerable interest. $N$-Heterocycles are known to form zwitterions with activated acetylene compounds such as dimethyl acetylenedicarboxylate..$^{5-11}$ These zwitterions intermediate can be trapped with a variety of electrophiles and proton donors, which is a novel protocol for the synthesis of heterocyclic compounds. ${ }^{5-13}$ Trapping of the zwitterion formed by the addition of isoquinoline to dimethyl acetylenedicarboxylate (DMAD) with electrophiles such as isocyanates, ${ }^{14} \mathrm{~N}$-tosylimines, ${ }^{15}$ quinines ${ }^{16}$ and electrophilic styrenes, ${ }^{17}$ has been recently used for the synthesis of different isoquinoline-fuzed heterocyclic systems. Reaction of electron-deficient

*e-mail: mohammadanary@yahoo.com acetylene esters with isoquinoline or quinoline has been also studied in the presence of organic acidic compounds. Reaction of quinoline-DMAD zwitterion with $\mathrm{C}-\mathrm{H}$ acidic compound indan-1, 3-dione was reported to afford pyrroloquinoline derivatives. ${ }^{18}$ Isoquinoline-DMAD zwitterion was also reported to react with $\mathrm{N}-\mathrm{H}$ acidic compounds such as pyrrole, indole ${ }^{19}$ and amide ${ }^{20}$ to afford substituted dihydroisoquinoline derivatives. In view of our interest in multicomponent reactions of nucleophiles with activated acetylenes and organic acidic compounds, ${ }^{21-23}$ we wish to report herein the results of our studies on the reaction of isoquinoline with acetylenedicarboxylic esters in the presence of $\mathrm{O}-\mathrm{H}$ acidic compounds such as 5,5-dimethyl-1,3-cyclohexanedione (Dimedone), 1,3-cyclohexanedione, 4-hydroxycoumarin or 4-hydroxy6-methylpyran-1-one.

\section{Results and Discussion}

In an initial experiment, the reaction of diethyl acetylenedicarboxylate (DEAD, 1) with dimedone (2) in the presence of isoquinoline $(\mathbf{3})$ in dichloromethane afforded ethyl 5,6,7,8-tetrahydro-7,7-dimethyl-2,5-dioxo$2 \mathrm{H}$-chromene-4-carboxylate (4a) in 95\% yield (Scheme 1). 
The product was characterized on the basis of spectroscopic data. In the ${ }^{1} \mathrm{H}$ NMR spectrum the two methylene groups was observed at $\delta 2.41$ and $2.72 \mathrm{ppm}$ as two single signals. The resonance signals due to the two ethoxycarbonyl groups appeared as a triplet at $\delta 1.34(\mathrm{~J} 7.2 \mathrm{~Hz})$ and a quartet at $\delta 4.38(J 7.2 \mathrm{~Hz})$, supporting the IR absorption at 1726 $\mathrm{cm}^{-1}$. A singlet was observed at $\delta 1.33$ for the two methyl groups. The olefinic proton appeared at $\delta 6.14 \mathrm{ppm}$ as a singlet. In the ${ }^{13} \mathrm{C}$ NMR spectrum twelve distinct signals were observed, which is consistent with the proposed structure. The structure and regiochemistry of compound 4a was unambiguously established by single crystal $\mathrm{X}$-ray analysis (Figure 1). The distinction between $\gamma$ - and $\delta$-lactonization products $\mathbf{3}$ and $\mathbf{4}$ was based on the X-ray crystallographic data. As shown in Scheme 1, the reaction was found to be applicable to DMAD. The spectral data for compound $\mathbf{4 b}$ were very similar to compound $\mathbf{4 a}$, with exceptions of the signals due to the alkoxycarbonyl groups.

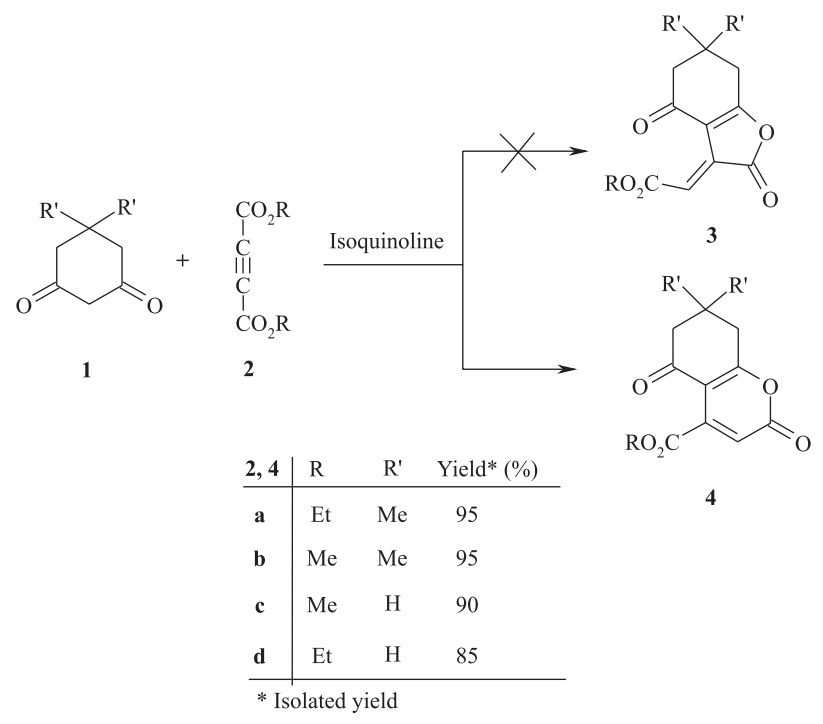

Scheme 1. Reaction between acetylenedicarboxylates and dimedone or1,3-cyclohexandione in the presence of isoquinoline.

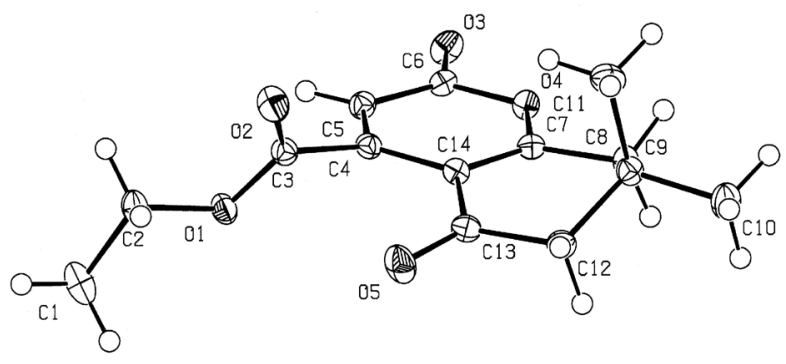

Figure 1. ORTEP representation of $\mathbf{4 a}$.

A reasonable mechanism for the formation of compound 4a is illustrated in Scheme 2. The zwitterion, formed from isoquinoline and DEAD (2) was protonated with dimedone to afford the cation $\mathbf{6}$ and the enolate ion 7. The anion $\mathbf{7}$ then underwent Michael addition to cation $\mathbf{6}$ to furnish the zwitterion $\mathbf{8}$, which was transformed into another zwitterion by an intramolecular proton transfer. Zwitterion intermediate 9 underwent lactonization to produce cation $\mathbf{1 0}$ and ethoxide anion. Finally, ethoxide anion absorbed a proton from the cation $\mathbf{1 0}$ and promoted the elimination of isoquinoline to furnish the product $\mathbf{4 a}$.

Under similar conditions, the reaction of isoquinoline with DMAD and 4-hydroxycoumarin (11) led to methyl 2,5-dihydro-2,5-dioxopyrano[3,2-c]chromene-4-carboxylate 12a in $90 \%$ yield (Scheme 3 ). In the ${ }^{1} \mathrm{H}$ NMR spectrum of compound 12a methoxy group was observed at $\delta 4.02 \mathrm{ppm}$ as a single signal. A singlet was observed at $\delta 6.41$ for the olefinic proton. The aromatic protons appeared at $\delta 6.27-8.12 \mathrm{ppm}$. In the ${ }^{13} \mathrm{C}$ NMR spectrum fourteen distinct signals were observed, which is consistent with the proposed structure.

As shown in Scheme 3, similar product 12b was obtained when DEAD was used as the activated acetylene. However, treatment of ditertiobutyl acetylenedicarboxylate (DTAD, 2c) with isoquinoline and 4-hydroxycoumarin (11), and separation of the reaction mixture by column chromatography afforded di-tert-butyl 2-(4-hydroxy-2-oxo$2 \mathrm{H}$-chromen-3-yl)fumarate $\mathbf{1 3}$ in $95 \%$ yield (Scheme 4).

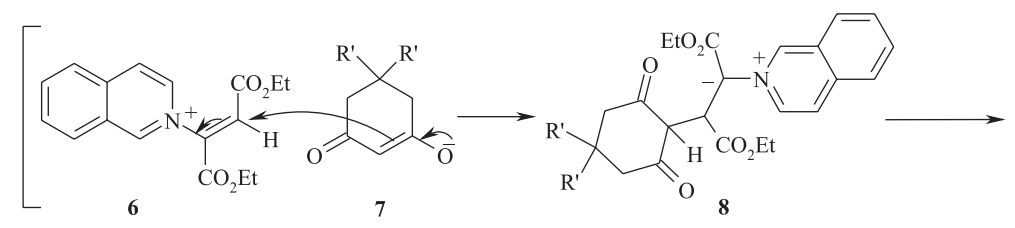

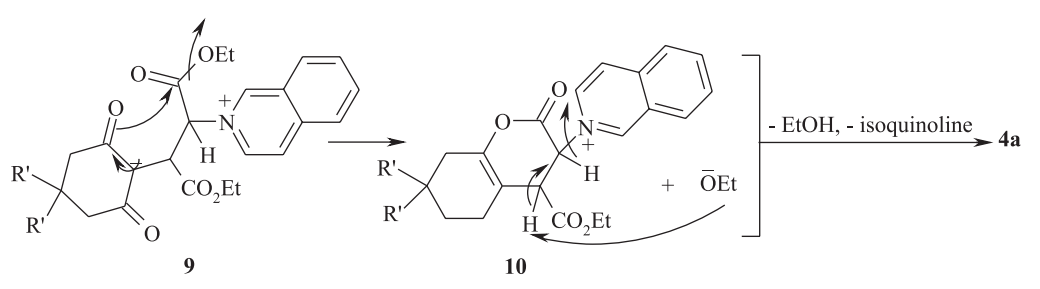

Scheme 2. Suggested mechanism for formation of compound $\mathbf{4 a}$. 
The ${ }^{1} \mathrm{H}$ NMR spectrum of compound $\mathbf{1 3}$ exhibited two sharp single signals at $\delta 1.43$ and $1.52 \mathrm{ppm}$ for two $t$-butyl groups. The olefinic proton appeared at $\delta 6.91 \mathrm{ppm}$ as a singlet. A broad singlet was observed for $\mathrm{O}-\mathrm{H}$ proton (removed by addition of $\mathrm{D}_{2} \mathrm{O}$ ) at $\delta 10.35 \mathrm{ppm}$. Aromatic protons were observed at $\delta$ 7.28-8.00 ppm. ${ }^{13} \mathrm{C}$ NMR spectrum showed sixteen resonances in agreement with the proposed structure.<smiles>O=c1cc(O)c2ccccc2o1</smiles>

11

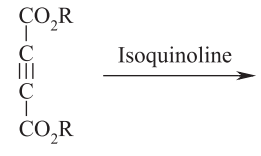

2

\begin{tabular}{r|cc}
$\mathbf{2 , 1 2}$ & $\mathrm{R}$ & Yield*(\%) \\
\hline $\mathbf{a}$ & $\mathrm{Me}$ & 90 \\
$\mathbf{b}$ & $\mathrm{Et}$ & 95 \\
\hline * Isolated yield
\end{tabular}

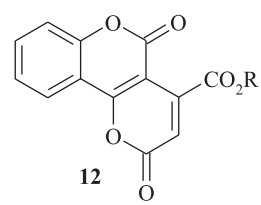

Scheme 3. Reaction between acetylenedicarboxylates and 4-hydroxycoumarin in the presence of isoquinoline.<smiles>O=c1cc(O)c2ccccc2o1</smiles>
1

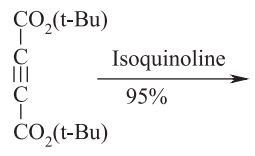

$2 \mathrm{c}$<smiles></smiles>

13
Scheme 4. Reaction between ditertiobutyl acetylenedicarboxylate and 4-hydroxycoumarin in the presence of isoquinoline.

The reaction of isoquinoline-DMAD zwitterion was also carried out towards 4-hydroxy-6-methylpyran-1-one (14) and methyl 2, 5-dihydro-7-methyl-2, 5-dioxopyrano [4, 3-b] pyran-4-carboxylate $\mathbf{1 5}$ was obtained in $90 \%$ yield (Scheme 5). The ${ }^{1} \mathrm{H}$ NMR spectrum of compound 15 exhibited four sharp single signals at $\delta 2.38(3 \mathrm{H}), 3.97$ $(3 \mathrm{H}), 6.24(1 \mathrm{H})$ and $6.33(1 \mathrm{H})$ for two methyl and two methine protons. ${ }^{13} \mathrm{C}$ NMR spectrum showed ten distinct resonances in agreement with the proposed structure.

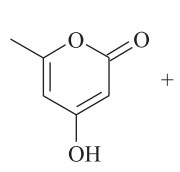

14

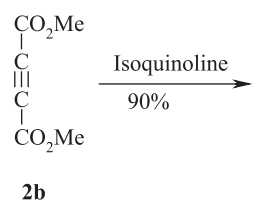

2b

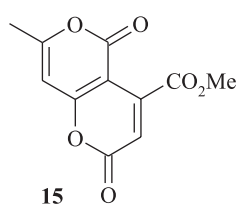

15
Scheme 5. Reaction between dimethyl acetylenedicarboxylate and 4-hydroxy-6-methylpyran-1-one in the presence of isoquinoline.

\section{Conclusions}

From the above results, we conclude that treatment of enolic systems such as dimedone, 4-hydroxycoumarin or 4-hydroxy-6-methylpyran-1-one with acetylenedicarboxylates and isoquinoline can lead to the formation of some fused heterocycles. The whole reaction can be considered as an addition reaction between acetylene derivative and enolic system, followed by a $\delta$-lactonization one, catalized by isoquinoline. The presented method has the advantage of being performed under neutral conditions and requires no activation or modification of the reagents.

\section{Experimental}

Melting points were determined with an electrothermal 9100 apparatus. Elemental analyses were performed using a Heraeus CHN-O-Rapid analyzer. Mass spectra were recorded on a Finnigan- MAT (San Jose, CA, USA) 8430 mass spectrometer operating at $70 \mathrm{eV}$. IR spectra were recorded on a Shimadzu (Tokyo, Japan) IR-470 spectrometer. ${ }^{1} \mathrm{H}$ and ${ }^{13} \mathrm{C}$ NMR spectra were recorded on BRUKER DRX-300 AVANCE spectrometer at 300.1 and $75.46 \mathrm{MHz}$, respectively. ${ }^{1} \mathrm{H}$ and ${ }^{13} \mathrm{C}$ NMR spectra were obtained on solution in $\mathrm{CDCl}_{3}$ using TMS as internal standard. The chemicals used in this work were purchased from Fluka (Buchs, Switzerland) and were used without further purification.

\section{General procedure}

To a magnetically stirred solution of the enolizable compound $(1 \mathrm{mmol})$ and isoquinoline $(1 \mathrm{mmol})$ in $10 \mathrm{~mL}$ acetone was added dropwise a mixture of acetylenedicarboxylates $(1 \mathrm{mmol})$ in $5 \mathrm{~mL}$ acetone at room temperature. The reaction mixture was then stirred for $24 \mathrm{~h}$. The solvent was removed under reduced pressure and the residue was purified by silica gel column chromatography using hexane-ethyl acetate as eluent to afford the pure title compounds.

Ethyl 5,6,7,8-tetrahydro-7,7-dimethyl-2,5-dioxo-2H-chromene -4-carboxylate ( $4 a)$

Colorless crystals; yield $0.25 \mathrm{~g}(95 \%)$, mp $96-98{ }^{\circ} \mathrm{C}$; $\operatorname{IR}(\mathrm{KBr}) v_{\max } / \mathrm{cm}^{-1}: 1760,1733(\mathrm{C}=\mathrm{O})$. MS $(\mathrm{m} / z, \%): 264$ $\left(\mathrm{M}^{+}, 8\right) .{ }^{1} \mathrm{H}$ NMR $\left(300.1 \mathrm{MHz}, \mathrm{CDCl}_{3}\right): \delta 1.13(6 \mathrm{H}, \mathrm{s}$, $\left.2 \mathrm{CH}_{3}\right), 1.34\left(3 \mathrm{H}, \mathrm{t},{ }^{3} \mathrm{~J}_{\mathrm{HH}} 7.2 \mathrm{~Hz} \mathrm{OCH}_{2} \mathrm{CH}_{3}\right), 2.41,2.72$ $\left(4 \mathrm{H}, 2 \mathrm{~s}, 2 \mathrm{CH}_{2}\right), 4.38\left(2 \mathrm{H}, \mathrm{q},{ }^{3} \mathrm{~J}_{\mathrm{HH}} 7.2 \mathrm{~Hz}, \mathrm{OCH}_{2} \mathrm{CH}_{3}\right), 6.14$ $(1 \mathrm{H}, \mathrm{s}, \mathrm{CH}) \mathrm{ppm} .{ }^{13} \mathrm{C} \mathrm{NMR}\left(75.46 \mathrm{MHz}, \mathrm{CDCl}_{3}\right): \delta 14.29$ $\left(\mathrm{OCH}_{2} \mathrm{CH}_{3}\right), 28.65(2 \mathrm{Me}), 32.95\left(\mathrm{C}(\mathrm{Me})_{2}\right), 42.54,51.09$, $\left(2 \mathrm{CH}_{2}\right) 62.99\left(\mathrm{OCH}_{2} \mathrm{CH}_{3}\right), 111.86(\mathrm{CH}), 112.19(\mathrm{O}-\mathrm{C}=\mathrm{C})$, 146.47( $\left.C-\mathrm{CO}_{2} \mathrm{Et}\right), 159.56(\mathrm{O}-\mathrm{C}=\mathrm{C}), 165.77,174.38,192.74$ $(3 \mathrm{C}=\mathrm{O})$ ppm. Anal.Calc. for $\mathrm{C}_{14} \mathrm{H}_{16} \mathrm{O}_{5}: \mathrm{C}, 63.63 ; \mathrm{H}, 6.10 \%$. Found: C, 63.81; H, 5.9\%.

\section{Crystal data for $4 a$}

Formula $\left(\mathrm{C}_{14} \mathrm{H}_{16} \mathrm{O}_{5}\right): \mathrm{Fw}=264.27$, trigonal, space group R-3, crystal dimensions $0.50 \times 0.49 \times 0.45 \mathrm{~mm}$, 
$a=22.5641(18) \AA, b=22.5641(18) \AA, c=13.2610(13) \AA$, $\alpha=90 \mathrm{deg}, \beta=90 \mathrm{deg}, \gamma=120 \mathrm{deg}$. V = 5847.1(9) $\AA^{3}, \mathrm{Z}=$ $18, \mathrm{D}_{\text {calc. }}=1.351 \mathrm{mg} \mathrm{m}^{-3}, 1.86^{\circ}<\theta<29.12^{\circ}, \mathrm{F}(000)=2520$; section of the reciprocal lattice: $-19 \leq \mathrm{h} \leq 30,-30 \leq \mathrm{k} \leq 26$, $-14 \leq 1 \leq 18$; of the 6231 reflections that were collected, 3384 were unique with $\mathrm{I}>2 \sigma(\mathrm{I})$; absorption coefficient $0.103 \mathrm{~mm}^{-1} \cdot \mathrm{R} 1=0.0398$ for $\mathrm{I}>2 \sigma(\mathrm{I})$ and $\mathrm{wR} 2=0.0993$; largest peak $\left(0.380 \mathrm{e}^{-3}\right)$ and hole $\left(-0.185 \mathrm{e}^{-3}\right)$.

Crystal data analyses: Stoe IPDSII two-circle diffractometer, $\mathrm{Mo}_{\mathrm{Ka}}$ radiation $(\lambda=0.71073 \AA)$, $\mathrm{T}=120(2) \mathrm{K}$, graphite monochromator; numerical absorption correction. Structure solution by direct methods using SHELXS and refinement by full-matrix least-squares on $\mathrm{F}^{2}$ using SHELXL of the X-STEP32 suite of programs, ${ }^{24}$ all non-hydrogen atoms were refined anisotropically.

The crystallographic data of $\mathbf{4 a}$ have been deposited with the Cambridge Crystallographic Data Centre as supplementary publication number CCDC-706464. Copies of the data can be obtained free of charge (http://www.ccdc. cam.ac.uk/ data_reques/cif, e-mail: data_request@ @ccdc. cam.ac.uk, or fax: +44-1223-336033).

Methyl 5,6,7,8-tetrahydro-7,7-dimethyl-2,5-dioxo-2Hchromene-4-carboxylate (4b)

Colorless crystals; yield $0.25 \mathrm{~g}(95 \%), \mathrm{mp} 96-98{ }^{\circ} \mathrm{C}$; $\operatorname{IR}(\mathrm{KBr}) \mathrm{v}_{\max } / \mathrm{cm}^{-1}: 1741,1679(\mathrm{C}=\mathrm{O})$. MS $(\mathrm{m} / \mathrm{z}, \%): 250$ $\left(\mathrm{M}^{+}, 10\right) .{ }^{1} \mathrm{H}$ NMR $\left(300.1 \mathrm{MHz}, \mathrm{CDCl}_{3}\right): \delta 1.19(6 \mathrm{H}, \mathrm{s}$, $\left.2 \mathrm{CH}_{3}\right), 2.46,2.86\left(4 \mathrm{H}, 2 \mathrm{~s}, 2 \mathrm{CH}_{2}\right), 3.85\left(3 \mathrm{H}, \mathrm{s}, \mathrm{OCH}_{3}\right), 6.25$ $(1 \mathrm{H}, \mathrm{s}, \mathrm{CH}) \mathrm{ppm} .{ }^{13} \mathrm{C}$ NMR $\left(75.46 \mathrm{MHz}, \mathrm{CDCl}_{3}\right): \delta 28.07$ $(2 \mathrm{Me}), 32.40\left(\mathrm{C}(\mathrm{Me})_{2}\right), 41.87,50.39,\left(2 \mathrm{CH}_{2}\right) 53.05\left(\mathrm{OCH}_{3}\right)$, $111.24(\mathrm{CH}), 111.67(\mathrm{O}-\mathrm{C}=C), 145.56\left(C-\mathrm{CO}_{2} \mathrm{Me}\right), 158.99$ $(\mathrm{O}-\mathrm{C}=\mathrm{C}), 165.76,174.16,192.46(3 \mathrm{C}=\mathrm{O}) \mathrm{ppm}$. Anal.Calc. for $\mathrm{C}_{13} \mathrm{H}_{14} \mathrm{O}_{5}: \mathrm{C}, 62.39 ; \mathrm{H}, 5.64 \%$. Found: $\mathrm{C}, 62.42 ; \mathrm{H}$, $5.73 \%$.

Methyl 5,6,7,8-tetrahydro-2,5-dioxo-2H-chromene-4carboxylate (4c)

Colorless oil; yield $0.19 \mathrm{~g}(90 \%)$. IR(KBr) $v_{\max } / \mathrm{cm}^{-1}$ : 1739, $1672(\mathrm{C}=\mathrm{O})$. MS $(\mathrm{m} / \mathrm{z}, \%): 222\left(\mathrm{M}^{+}, 8\right) .{ }^{1} \mathrm{H}$ NMR $\left(300.1 \mathrm{MHz}, \mathrm{CDCl}_{3}\right): \delta 2.21\left(2 \mathrm{H}, \mathrm{m}, \mathrm{CH}_{2}\right), 2.61(2 \mathrm{H}, \mathrm{t}$, $\left.\mathrm{CH}_{2},{ }^{3} J_{\mathrm{HH}} 6.3 \mathrm{~Hz}\right), 2.92\left(2 \mathrm{H}, \mathrm{t}, \mathrm{CH}_{2},{ }^{3} J_{\mathrm{HH}} 6.3 \mathrm{~Hz}\right), 3.97(3 \mathrm{H}$, $\left.\mathrm{s}, \mathrm{OCH}_{3}\right), 6.23(1 \mathrm{H}, \mathrm{s}, \mathrm{CH}) \mathrm{ppm} .{ }^{13} \mathrm{C}$ NMR $(75.46 \mathrm{MHz}$, $\left.\mathrm{CDCl}_{3}\right): \delta 19.87,28.55$ and $36.53\left(3 \mathrm{CH}_{2}\right), 53.24\left(\mathrm{OCH}_{3}\right)$, $112.16(\mathrm{CH}), 112.45(\mathrm{O}-\mathrm{C}=C), 145.92\left(C-\mathrm{CO}_{2} \mathrm{Me}\right), 158.78$ $(\mathrm{O}-\mathrm{C}=\mathrm{C}), 165.9,175.55,192.42(3 \mathrm{C}=\mathrm{O}) \mathrm{ppm}$. Anal.Calc. for $\mathrm{C}_{11} \mathrm{H}_{10} \mathrm{O}_{5}: \mathrm{C}, 59.46 ; \mathrm{H}, 4.54 \%$. Found: C, 59.52; H, $4.41 \%$.

Ethyl 5,6,7,8-tetrahydro-2,5-dioxo-2H-chromene-4carboxylate $(\mathbf{4 d})$

Colorless oil; yield $0.20 \mathrm{~g}(85 \%)$. IR(KBr) $v_{\max } / \mathrm{cm}^{-1}: 1760$, $1686(\mathrm{C}=\mathrm{O}), \mathrm{MS}(\mathrm{m} / \mathrm{z}, \%): 236\left(\mathrm{M}^{+}, 15\right) .{ }^{1} \mathrm{HNMR}(300.1 \mathrm{MHz}$,
$\left.\mathrm{CDCl}_{3}\right): \delta 1.37\left(3 \mathrm{H}, \mathrm{t},{ }^{3} \mathrm{~J}_{\mathrm{HH}} 7.3 \mathrm{~Hz}, \mathrm{OCH}_{2} \mathrm{CH}_{3}\right), 2.18(2 \mathrm{H}, \mathrm{m}$, $\left.\mathrm{CH}_{2}\right), 2.59\left(2 \mathrm{H}, \mathrm{t}, \mathrm{CH}_{2},{ }^{3} \mathrm{~J}_{\mathrm{HH}} 6.3 \mathrm{~Hz}\right), 2.89\left(2 \mathrm{H}, \mathrm{t}, \mathrm{CH}_{2},{ }^{3} \mathrm{~J}_{\mathrm{HH}} 6.3\right.$ $\mathrm{Hz}), 4.40\left(2 \mathrm{H}, \mathrm{q},{ }^{3} \mathrm{~J}_{\mathrm{HH}} 7.3 \mathrm{~Hz}, \mathrm{OCH}_{2} \mathrm{CH}_{3}\right), 6.17(1 \mathrm{H}, \mathrm{s}, \mathrm{CH}) \mathrm{ppm}$. ${ }^{13} \mathrm{C} \mathrm{NMR}\left(75.46 \mathrm{MHz}, \mathrm{CDCl}_{3}\right): \delta 13.86\left(\mathrm{OCH}_{2} \mathrm{CH}_{3}\right), 19.87$, 28.54 and $36.52\left(3 \mathrm{CH}_{2}\right), 62.55\left(\mathrm{OCH}_{2} \mathrm{CH}_{3}\right), 111.91(\mathrm{CH})$, 112.43(O-C=C), $146.32\left(C-\mathrm{CO}_{2} \mathrm{Et}\right), 158.87(\mathrm{O}-C=\mathrm{C})$, 165.41, 175.46, $192.43(3 \mathrm{C}=\mathrm{O}) \mathrm{ppm}$. Anal.Calc. for $\mathrm{C}_{12} \mathrm{H}_{12} \mathrm{O}_{5}$ : C, 61.01; H, 5.12\%. Found: C, 61.18; H, 5.28\%.

Methyl 2,5-dihydro-2,5-dioxopyrano[3,2-c]chromene-4carboxylate (12a)

Colorless crystals; yield $0.24 \mathrm{~g}(90 \%), \mathrm{mp} 191{ }^{\circ} \mathrm{C}$ dec.; $\operatorname{IR}(\mathrm{KBr}) v_{\text {max }} / \mathrm{cm}^{-1}: 1758,1715(\mathrm{C}=\mathrm{O})$. MS $(\mathrm{m} / \mathrm{z}, \%)$ : $272\left(\mathrm{M}^{+}, 6\right) .{ }^{1} \mathrm{H} \mathrm{NMR}\left(300.1 \mathrm{MHz}, \mathrm{CDCl}_{3}\right): \delta 4.02(3 \mathrm{H}, \mathrm{s}$, $\left.\mathrm{OCH}_{3}\right), 6.41(1 \mathrm{H}, \mathrm{s}, \mathrm{CH}), 7.42-8.12(4 \mathrm{H}, \mathrm{m}$, arom $) \mathrm{ppm} .{ }^{13} \mathrm{C}$ NMR $\left(75.46 \mathrm{MHz}, \mathrm{CDCl}_{3}\right): \delta 54.04\left(\mathrm{OCH}_{3}\right), 101.22(\mathrm{CH})$, 113.16, 113.58, 117.83, 124.31, 125.83, 135.67, 147.02, 154.01, 157.35 (c, arom), 157.51, 163.13, 164.99 (3C=O) ppm. Anal.Calc. for $\mathrm{C}_{14} \mathrm{H}_{8} \mathrm{O}_{6}: \mathrm{C}, 61.77 ; \mathrm{H}, 2.96 \%$. Found: C, $61.89 ; \mathrm{H}, 2.81 \%$.

Ethyl 2,5-dihydro-2,5-dioxopyrano[3,2-c]chromene-4carboxylate (12b)

Colorless crystals; yield $0.25 \mathrm{~g}(90 \%), \mathrm{mp} 180{ }^{\circ} \mathrm{C} \mathrm{dec}$; $\operatorname{IR}(\mathrm{KBr}) \mathrm{v}_{\max } / \mathrm{cm}^{-1}: 1739,1726(\mathrm{C}=\mathrm{O})$. MS $(\mathrm{m} / \mathrm{z}, \%): 286$ $\left(\mathrm{M}^{+}, 5\right) .{ }^{1} \mathrm{H}$ NMR $\left(300.1 \mathrm{MHz}, \mathrm{CDCl}_{3}\right): \delta 1.42\left(3 \mathrm{H}, \mathrm{t},{ }^{3} J_{\mathrm{HH}}\right.$ $\left.7.2 \mathrm{~Hz} \mathrm{OCH}_{2} \mathrm{CH}_{3}\right), 4.49\left(2 \mathrm{H}, \mathrm{q},{ }^{3} \mathrm{JHH}_{\mathrm{HH}} 7.2 \mathrm{~Hz}, \mathrm{OCH}_{2} \mathrm{CH}_{3}\right)$, $6.40(1 \mathrm{H}, \mathrm{s}, \mathrm{CH}), 7.42-8.12(4 \mathrm{H}, \mathrm{m}$, arom $) \mathrm{ppm} .{ }^{13} \mathrm{C}$ NMR (75.46 MHz, $\left.\mathrm{CDCl}_{3}\right): \delta 13.69\left(\mathrm{OCH}_{2} \mathrm{CH}_{3}\right), 62.53$ $\left(\mathrm{OCH}_{2} \mathrm{CH}_{3}\right), 102.11(\mathrm{CH}), 113.41,113.63,117.51,124.04$, 125.70, 135.43, 147.27, 154.04, 157.22 (c, arom), 157.60, 162.74, $164.39(3 \mathrm{C}=\mathrm{O}) \mathrm{ppm}$. Anal. Calc. for $\mathrm{C}_{15} \mathrm{H}_{10} \mathrm{O}_{6}: \mathrm{C}$, 62.94; H, 3.52\%. Found: C, 62.83; H, 3.61\%.

\section{Ditertbutyl 2-(4-hydroxy-2-oxo-2H-chromen-3-yl)fumarate} (13)

Colorless crystals; yield $0.36 \mathrm{~g}(95 \%), \mathrm{mp} 72-73{ }^{\circ} \mathrm{C}$; $\operatorname{IR}(\mathrm{KBr}) v_{\max } / \mathrm{cm}^{-1}: 1712,1660(\mathrm{C}=\mathrm{O})$. MS $(\mathrm{m} / \mathrm{z}, \%): 388$ $\left(\mathrm{M}^{+}, 7\right) .{ }^{1} \mathrm{H}$ NMR $\left(300.1 \mathrm{MHz}, \mathrm{CDCl}_{3}\right): \delta 1.43,1.52$ $(18 \mathrm{H}, 2 \mathrm{~s}, 2 \mathrm{t}-\mathrm{Bu}), 6.91(1 \mathrm{H}, \mathrm{s}, \mathrm{CH}), 7.28-8.00$ (4H arom), $10.35(1 \mathrm{H}$, broad singlet, $\mathrm{OH}) \mathrm{ppm} .{ }^{13} \mathrm{C}$ NMR $(75.46 \mathrm{MHz}$, $\left.\mathrm{CDCl}_{3}\right): \delta 29.72\left(2 \mathrm{C}\left(\mathrm{CH}_{3}\right)_{3}\right), 83.61,84.33\left(2 \mathrm{C}\left(\mathrm{CH}_{3}\right)_{3}\right)$, 101.96, 116.40, 116.43, 124.08, 124.48, 132.08, 132.77, 136.37, 152.93, 162.05 (aromatic and olefinic carbons), 162.15, 166.37, $167.41(3 \mathrm{C}=\mathrm{O}) \mathrm{ppm}$. Anal.Calc. for $\mathrm{C}_{21} \mathrm{H}_{24} \mathrm{O}_{7}: \mathrm{C}, 64.94 ; \mathrm{H}, 6.23 \%$. Found: $\mathrm{C}, 65.09 ; \mathrm{H}, 6.17 \%$.

Methyl 2, 5-dihydro-7-methyl-2, 5-dioxopyrano [4, 3-b] pyran-4-carboxylate (15)

Colorless crystals; yield $0.21 \mathrm{~g}(90 \%)$, mp $96-98^{\circ} \mathrm{C}$; $\mathrm{IR}(\mathrm{KBr}) \mathrm{v}_{\max } / \mathrm{cm}^{-1}: 1756,1721(\mathrm{C}=\mathrm{O})$. MS $(\mathrm{m} / \mathrm{z}, \%): 236$ 
$\left(\mathrm{M}^{+}, 7\right) .{ }^{1} \mathrm{H} \mathrm{NMR}\left(300.1 \mathrm{MHz}, \mathrm{CDCl}_{3}\right): \delta 2.38\left(3 \mathrm{H}, \mathrm{s}, \mathrm{CH}_{3}\right)$, $3.97\left(3 \mathrm{H}, \mathrm{s}, \mathrm{OCH}_{3}\right), 6.24,6.33(2 \mathrm{H}, 2 \mathrm{~s}, 2 \mathrm{CH}) \mathrm{ppm} .{ }^{13} \mathrm{C} \mathrm{NMR}$ $\left(75.46 \mathrm{MHz}, \mathrm{CDCl}_{3}\right): \delta 19.88\left(\mathrm{CH}_{3}\right), 52.87\left(\mathrm{OCH}_{3}\right), 99.48$, 111.56, 146.64, 157.92, 157.99 (olefinic carbons) 164.94, 167.83, $167.88(3 \mathrm{C}=\mathrm{O}) \mathrm{ppm}$. Anal.Calc. for $\mathrm{C}_{11} \mathrm{H}_{8} \mathrm{O}_{6}: \mathrm{C}$, 55.94; H, 3.41\%. Found: C, 55.81; H, 3.53\%.

\section{Supplementary Information}

Supplementary data is available free of charge at http://jbcs.sbq.org.br, as PDF file.

\section{References}

1. Wulf, H.; Rauer, H.; During, T.; Hanselmann, C.; Ruff, K.; Wrisch, A.; Grissmer, S.; Hansel, W.; J. Med. Chem. 1998, 41, 4542 .

2. Spino, C.; Dodier, M.; Sotheeswaren, S.; Bioorg. Med. Chem. Lett. 1998, 8, 3475.

3. Crow, F. W.; Duholke, W. K.; Farley, K. A.; Hadden, C. E.; Hahn, D. A.; Kaluzny, B. D.; Mallory, C. S.; Martin, G. E.; Smith, R. F.; Thamann, T. J.; J. Heterocycl. Chem. 1999, 36, 365.

4. Barker, W. M.; Hermodson, M. A.; Link, K. P.; J. Med. Chem. 1971, 14, 167.

5. Acheson, R. M.; Woollard, J.; J. Chem. Soc., Perkin Trans. 1 1975, 438.

6. Acheson, R. M.; Gagan, J. M. F.; Taylor, G. A.; J. Chem. Soc. 1963, 1903.

7. Acheson, R. M.; Plunkett, A. O.; J. Chem. Soc. 1964, 2676.

8. Huisgen, R.; Morikawa, M.; Herbig, K.; Brunn, E.; Chem. Ber. 1967, 100, 1094.

9. Winterfeldt, E.; Schumann, D.; Dillinger, H. J.; Chem. Ber. 1969, 102, 1656.
10. Dillinger, H. J.; Fengler, G.; Schumann, D.; Winterfeldt, E.; Tetrahedron 1974, 30, 2553.

11. Yavari, I.; Moradi, L.; Tetrahedron Lett. 2006, 47, 1627.

12. Yavari, I.; Moradi, L.; Mokhtarporyani-Sanandaj, A.; Mirzaei, A.; Helv. Chim. Acta 2007, 90, 392.

13. Yavari, I.; Mokhtarporyani-Sanandaj, A.; Moradi, L.; Tetrahedron Lett. 2007, 48, 6709.

14. Adib, M.; Mollahosseini, M.; Yavari, H.; Sayahi, M. H.; Bijanzadeh, H. R.; Synthesis 2004, 6, 861.

15. Nair, V.; Sreekanth, A. R.; Abhilash, N.; Bhadbhade, M. M.; Gonnade, R. C.; Org. Lett. 2002, 4, 575.

16. Nair, V.; Sreekanth, A .R.; Biju, A. T.; Rath, N. P.; Tetrahedron Lett. 2003, 44, 729.

17. Nair,V.; Remadevi, B.; Varma, L. R.; Tetrahedron Lett. 2005, 46, 5333.

18. Nair, V.; Devipriya, S.; Suresh, E.; Synthesis 2008, 7, 1065.

19. Yadav, J. S.; Subba Reddy, B. V.; Nagendra Nath, Y.; Gupta Manoj, K.; Tetrahedron Lett. 2008, 49, 2815.

20. Yavari, I.; Ghazanfarpour-Darjani, M.; Sabbaghan, M.; Hossaini, Z.; Tetrahedron Lett. 2007, 48, 3749.

21. Anary-Abbasinejad, M.; Anaraki-Ardakani, H.; Ghanea, F.; Monatsh. Chem. 2009, 140, 397.

22. Anaraki-Ardakani, H.; Sadeghian, S.; Rastegari, F.; Hassanabadi, A.; Anary-Abbasinejad, M.; Synth. Commun. 2008, 38, 1990.

23. Anary-Abbasinejad, M.; Anaraki-Ardakani, H.; HosseiniMehdiabad, H.; Phosphorus, Sulfur Silicon Relat. Elem. 2008, 183,1440 .

24. Stoe \& Cie; X-STEP32, Version 1.07b, Crystallographic package, Stoe \& Cie GmbH: Darmstadt, Germany, 2000.

Received: March 17, 2009

Web Release Date: November 26, 2009 
Isoquinoline-Catalyzed Reaction between 4-Hydroxycoumarin or 4-Hydroxy-6-methylpyran-1-one and Dialkyl Acetylene Dicarboxylates: Synthesis of Coumarin and Pyranopyrane Derivatives

\section{Mohammad Anary-Abbasinejad, ${ }^{*, a}$ Hossein Anaraki-Ardakani, ${ }^{a}$ Mohammad Hossein Mosslemin ${ }^{a}$ and Hamid Reza Khavasi ${ }^{b}$}

${ }^{a}$ Department of Chemistry, Islamic Azad University, Yazd Branch, PO Box 89195-155, Yazd, Iran

${ }^{b}$ Department of Chemistry, Shahid Beheshti University, PO Box 1983963113, Tehran, Iran
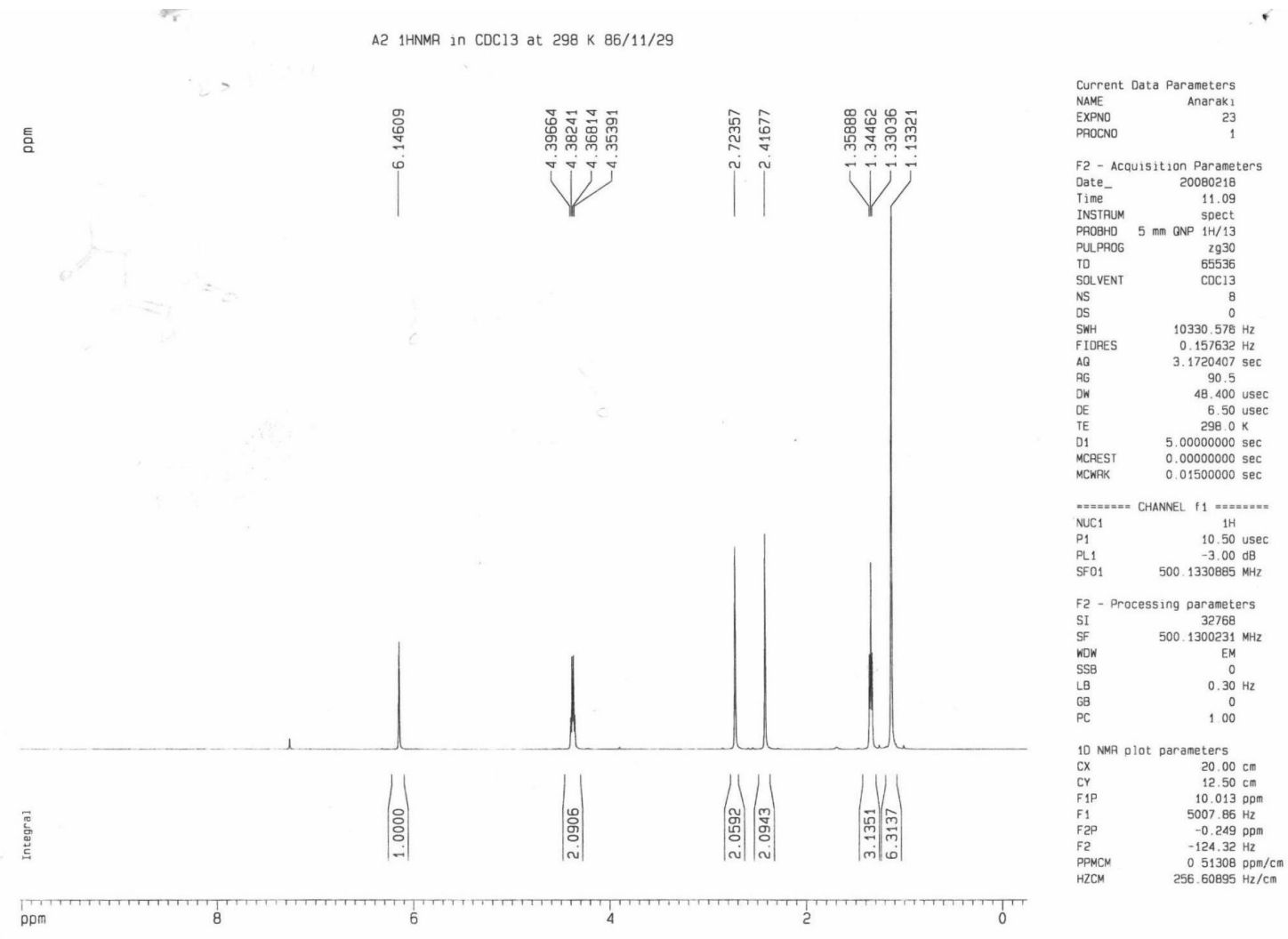

Figure S1. ${ }^{1} \mathrm{H}$ NMR spectrum of compound $\mathbf{4 a}$. 
A2 13 CNMR in $\mathrm{CDC} 3$ at $298 \mathrm{~K} 86 / 11 / 29$

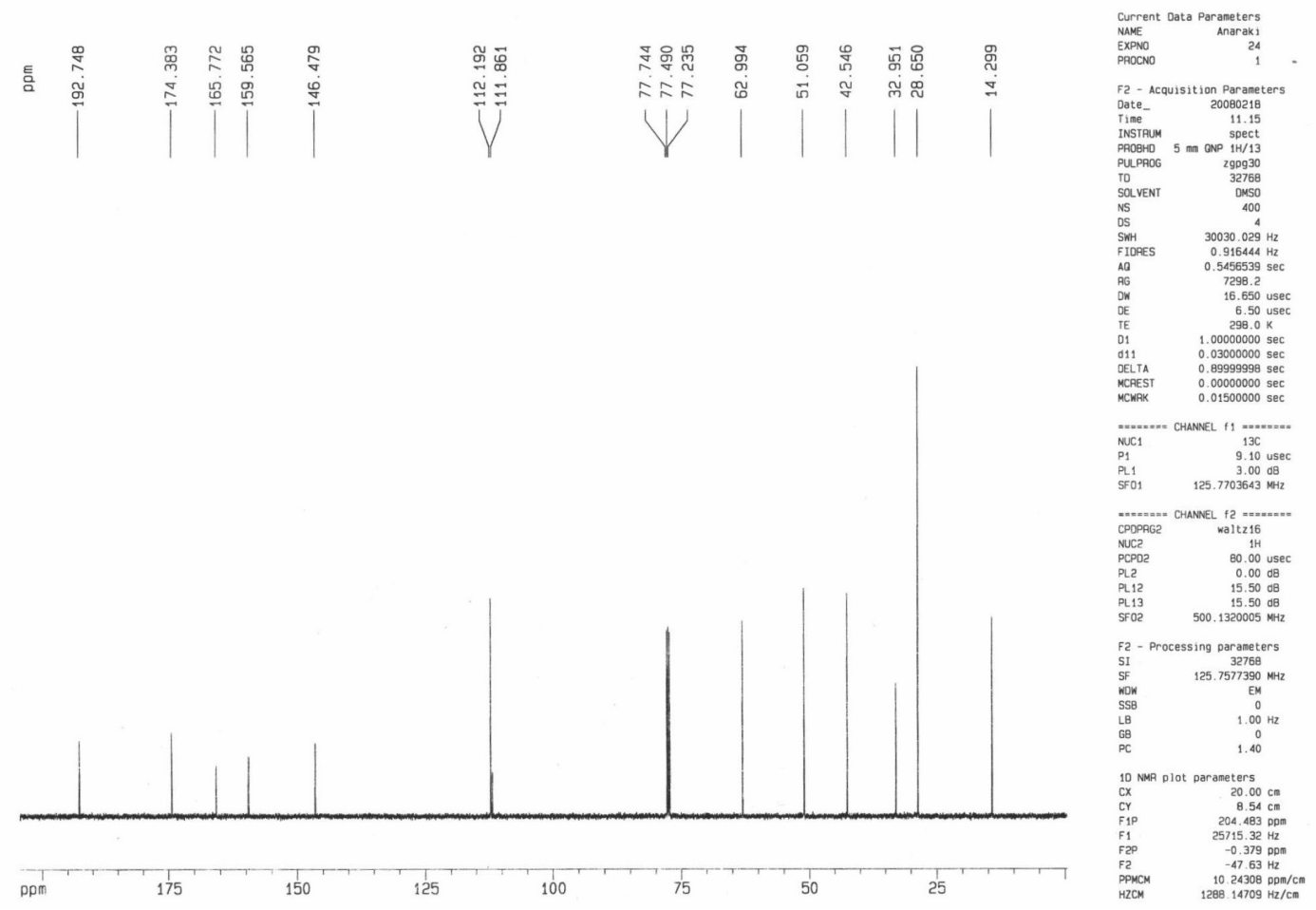

Figure S2. ${ }^{13} \mathrm{C}$ NMR spectrum of compound $\mathbf{4 a}$.
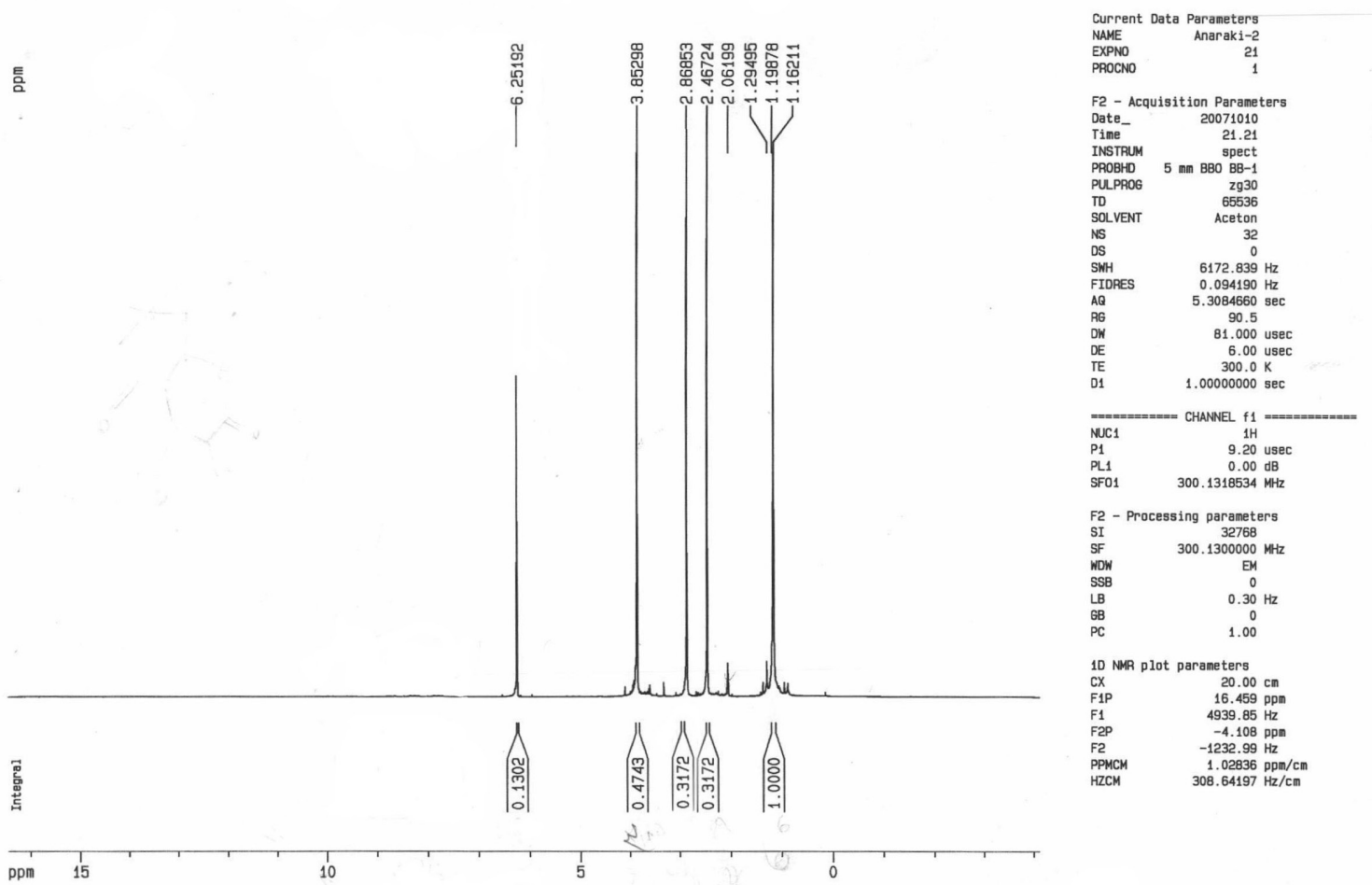

Figure S3. ${ }^{1} \mathrm{H}$ NMR spectrum of compound $\mathbf{4 b}$. 


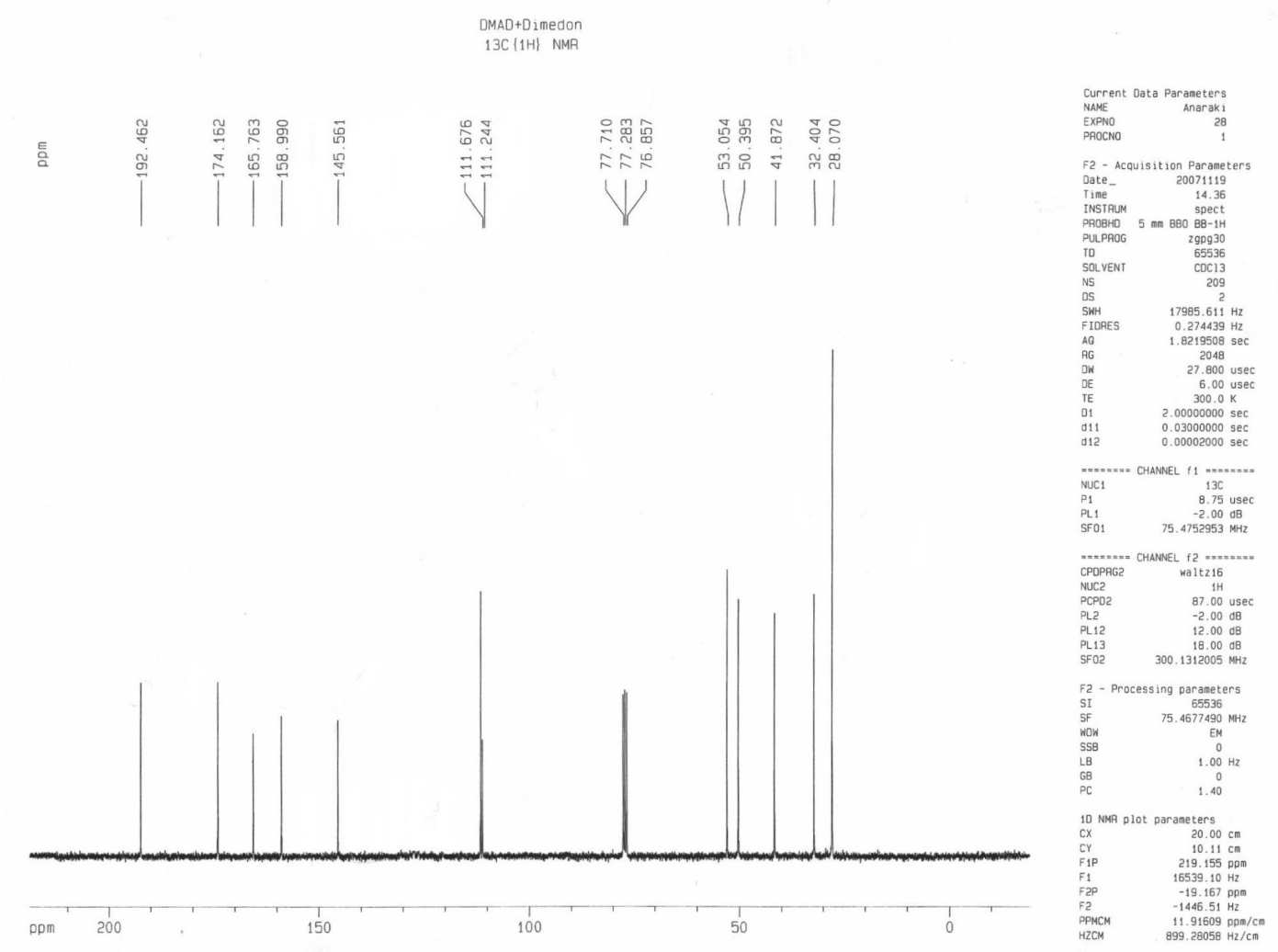

Figure S4. ${ }^{13} \mathrm{C}$ NMR spectrum of compound $\mathbf{4 b}$.

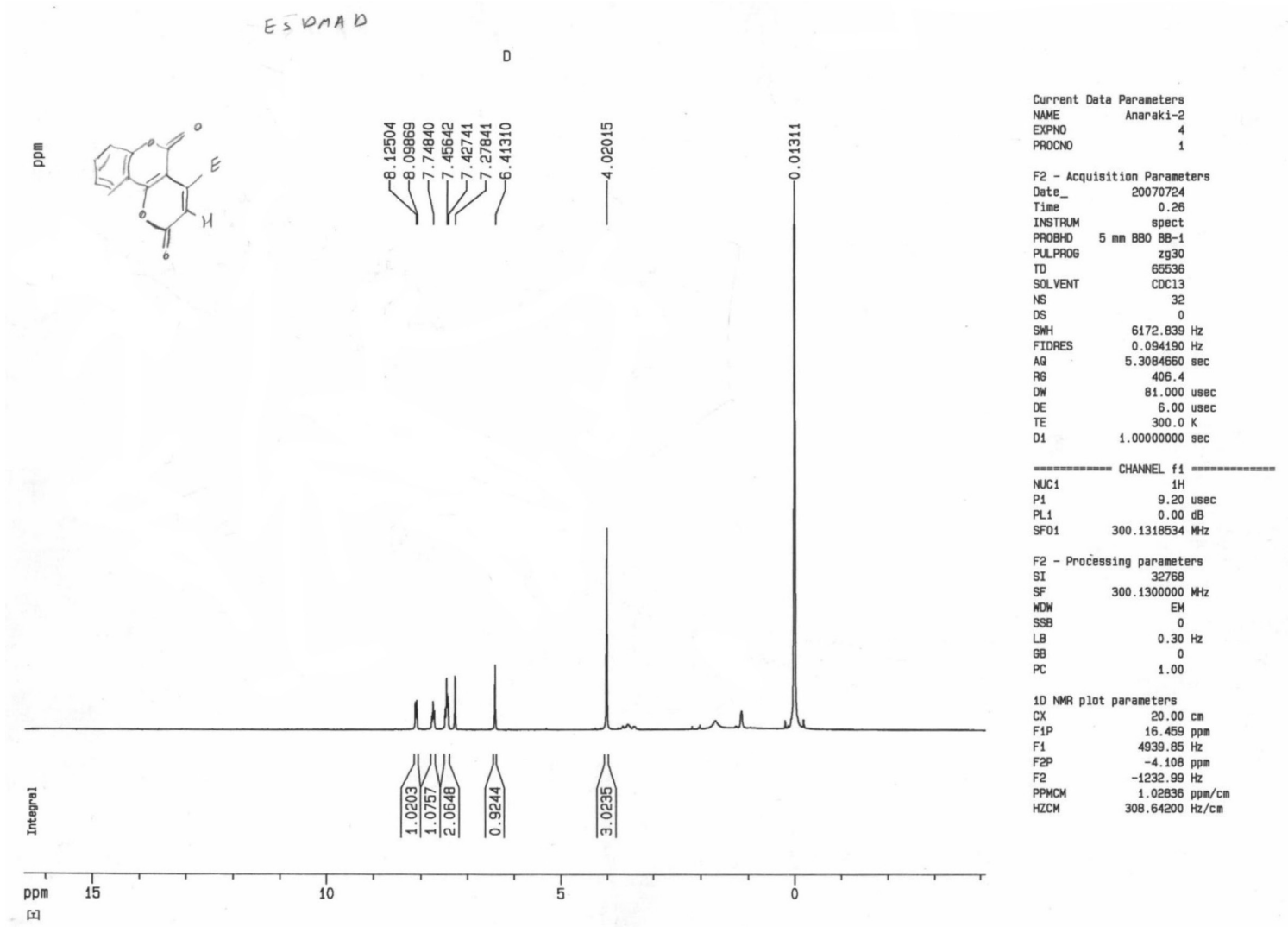

Figure S5. 'H NMR spectrum of compound 12a. 


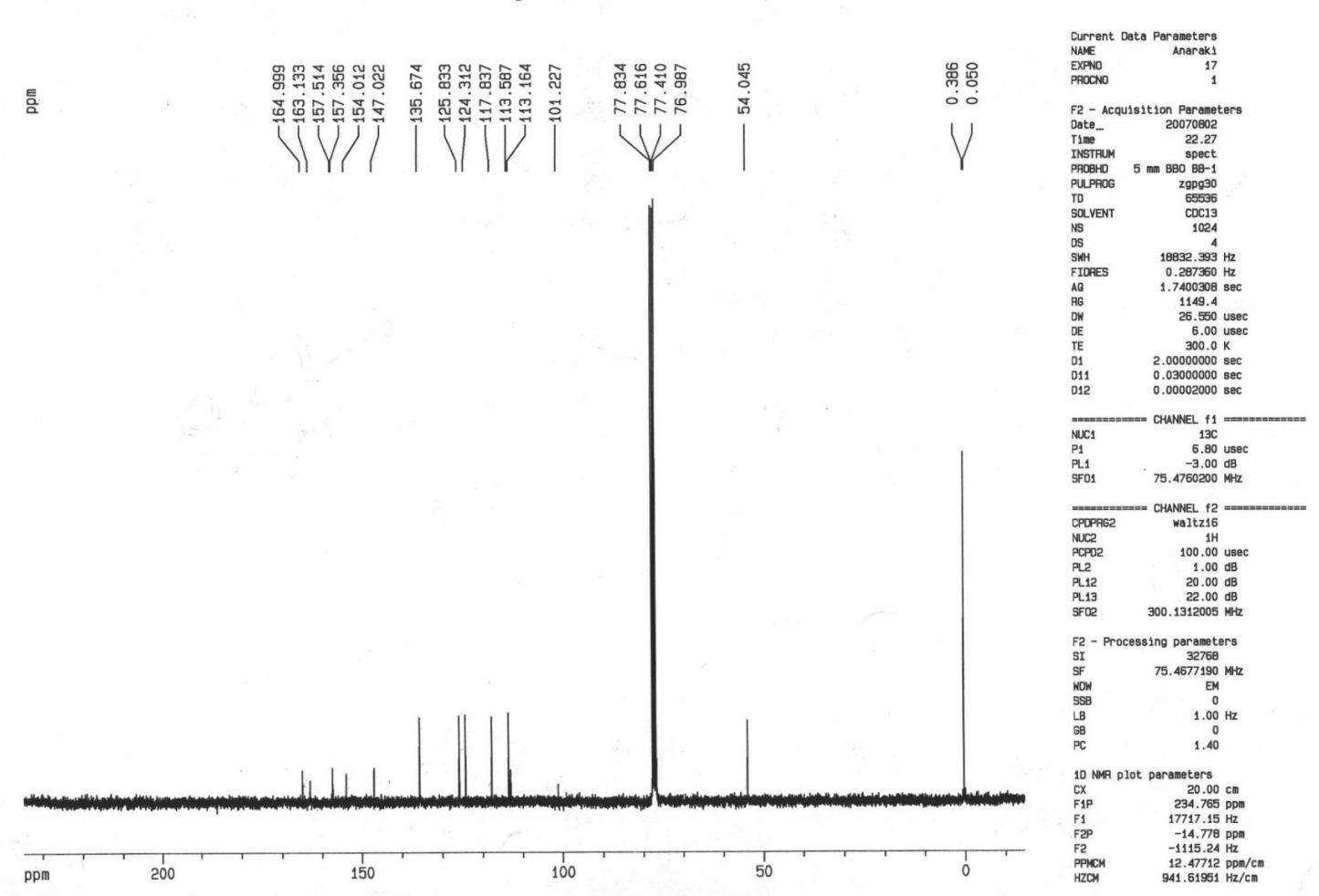

Figure S6. ${ }^{13} \mathrm{C}$ NMR spectrum of compound 12a.

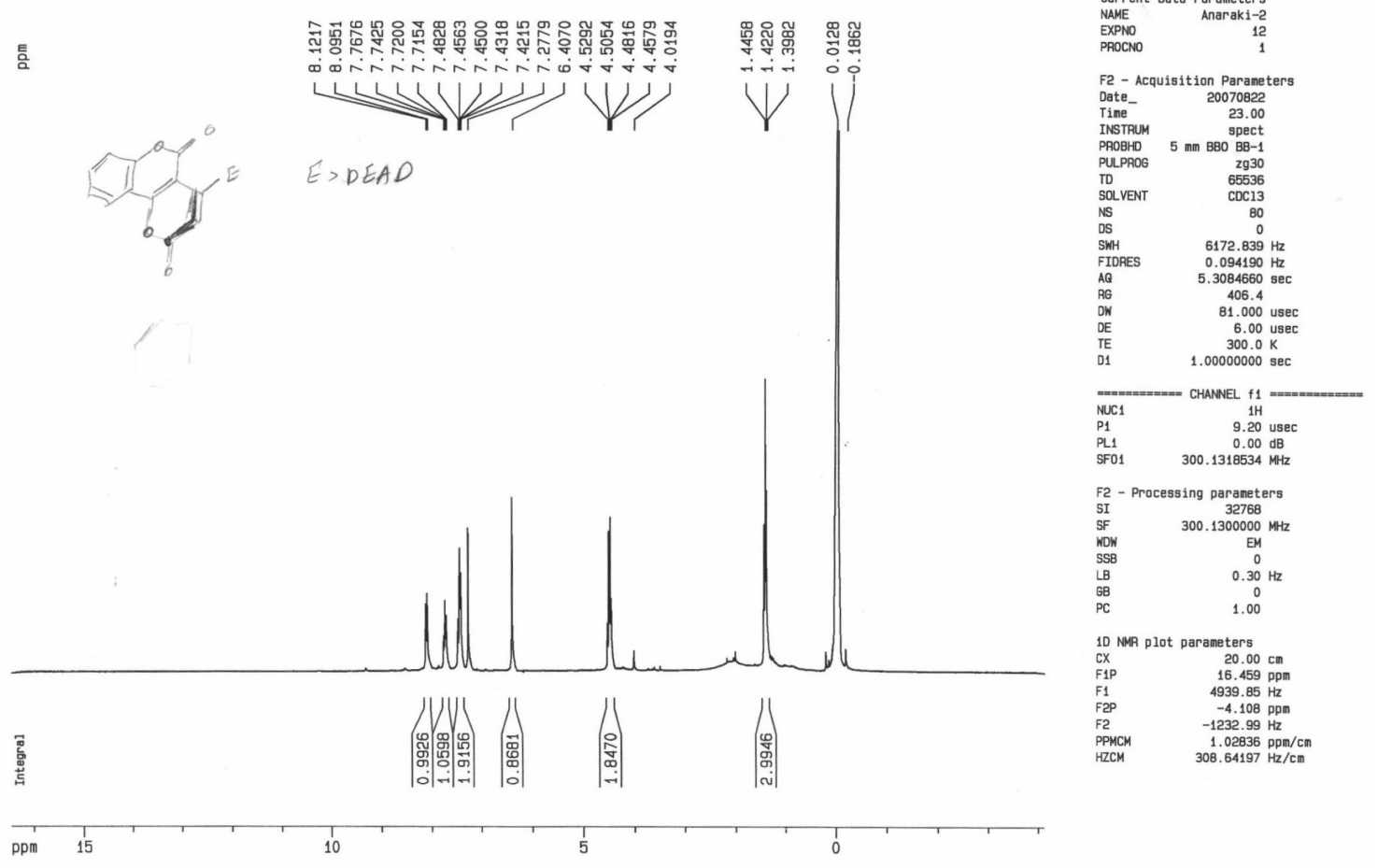

Figure S7. ${ }^{1} \mathrm{H}$ NMR spectrum of compound $\mathbf{1 2 b}$. 

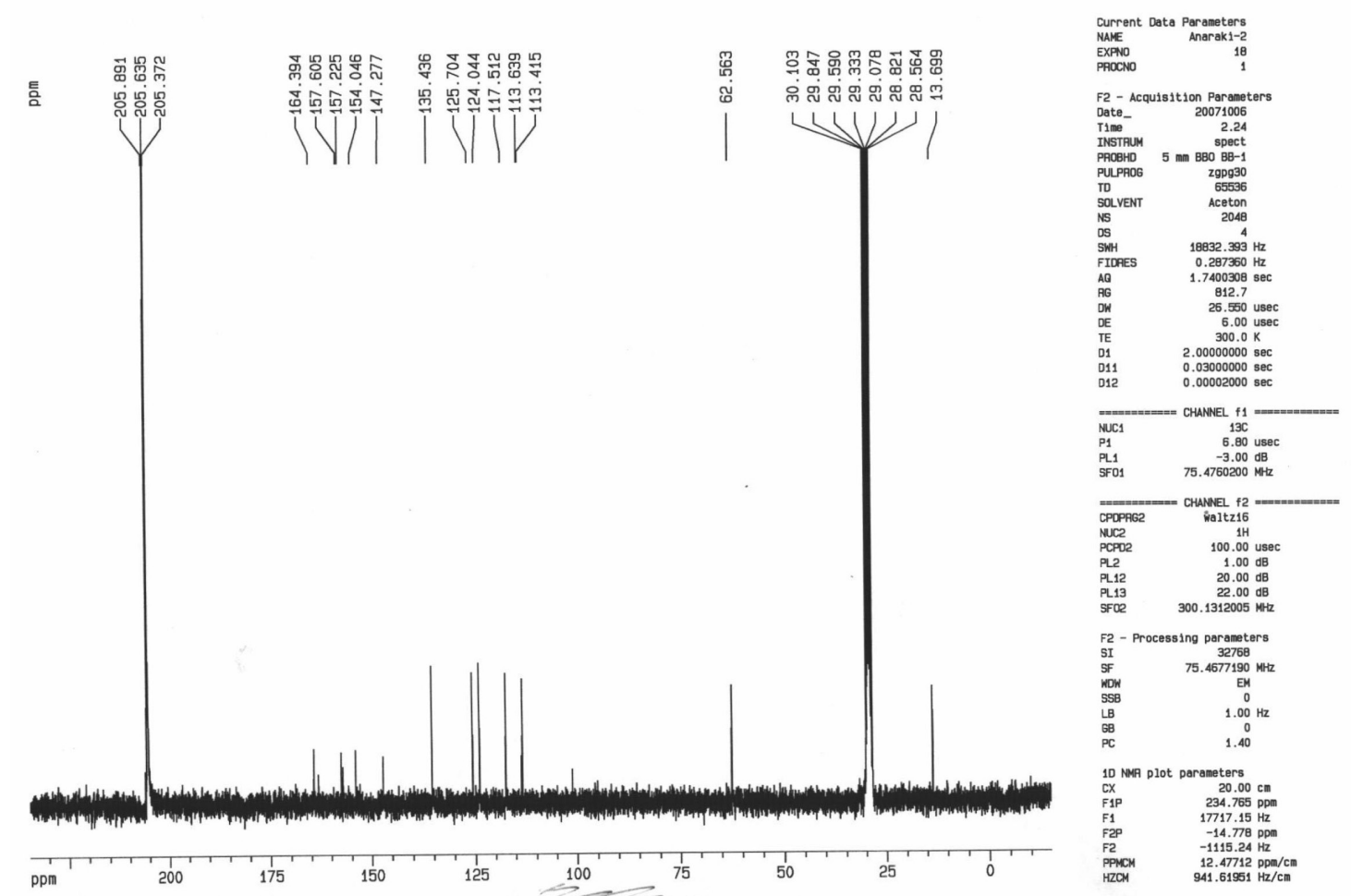

Figure S8. ${ }^{13} \mathrm{C}$ NMR spectrum of compound $\mathbf{1 2 b}$.
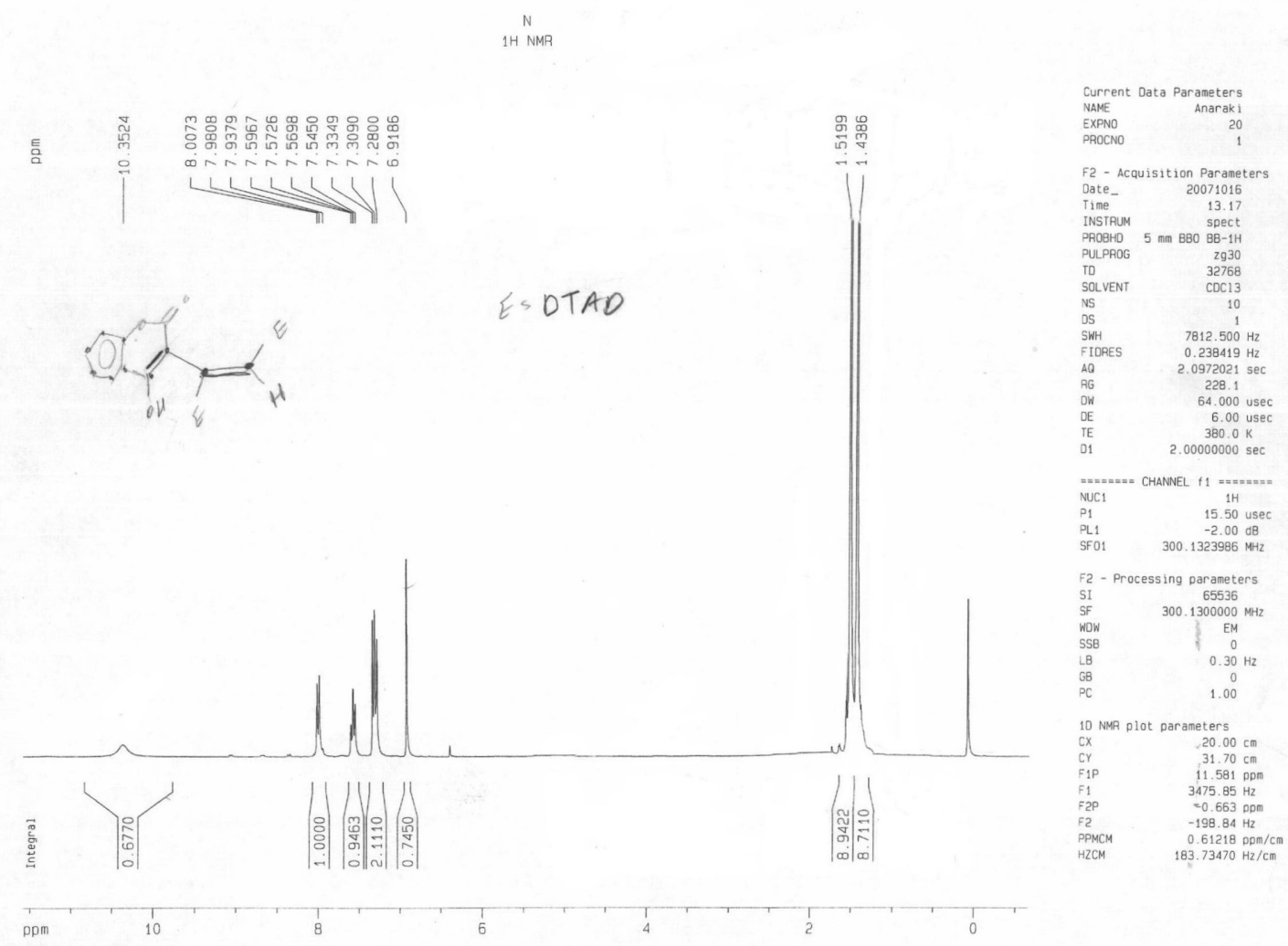

Figure S9. ${ }^{1} \mathrm{H}$ NMR spectrum of compound 13. 


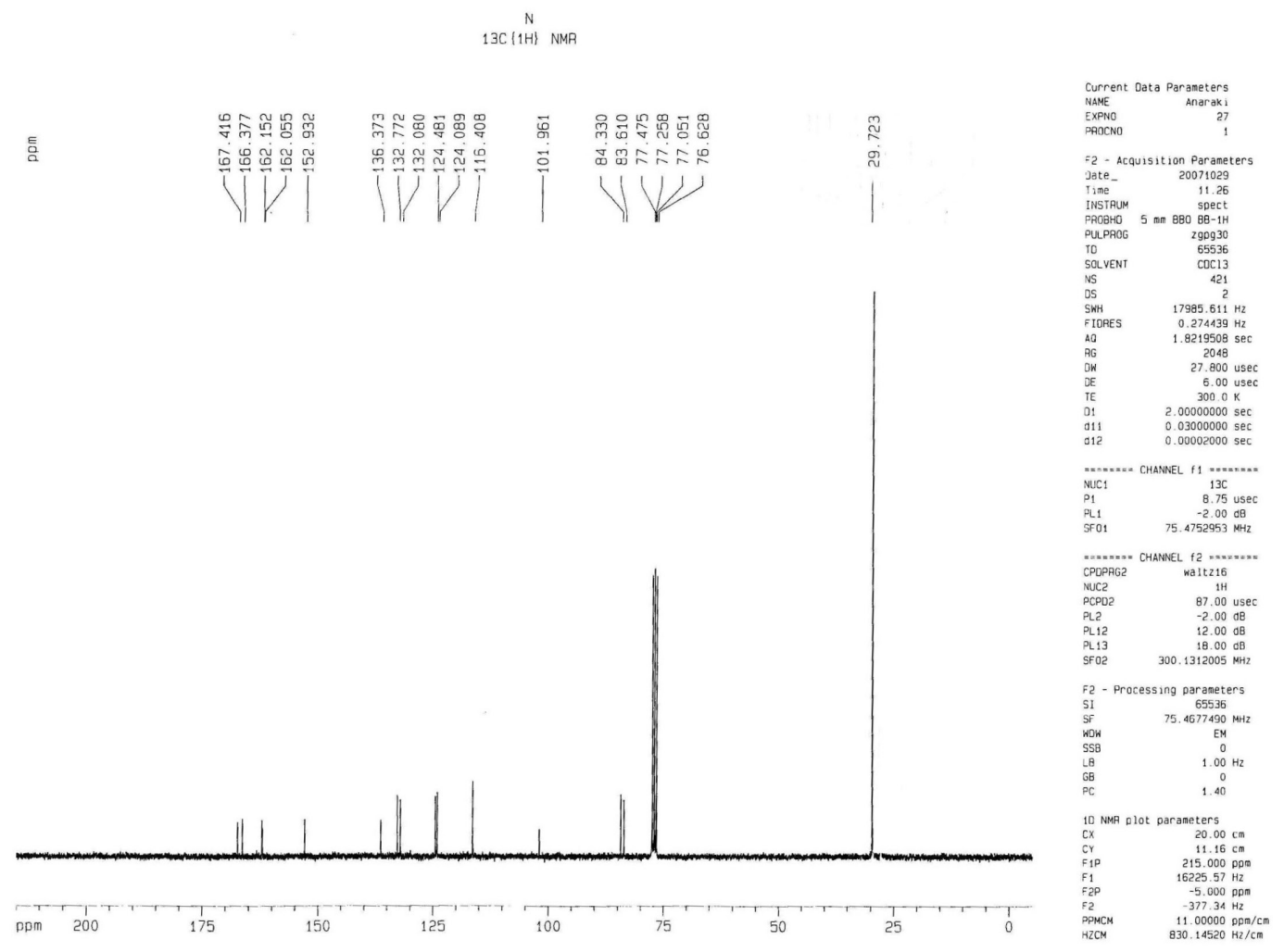

Figure S10. ${ }^{13} \mathrm{C}$ NMR spectrum of compound $\mathbf{1 3}$.

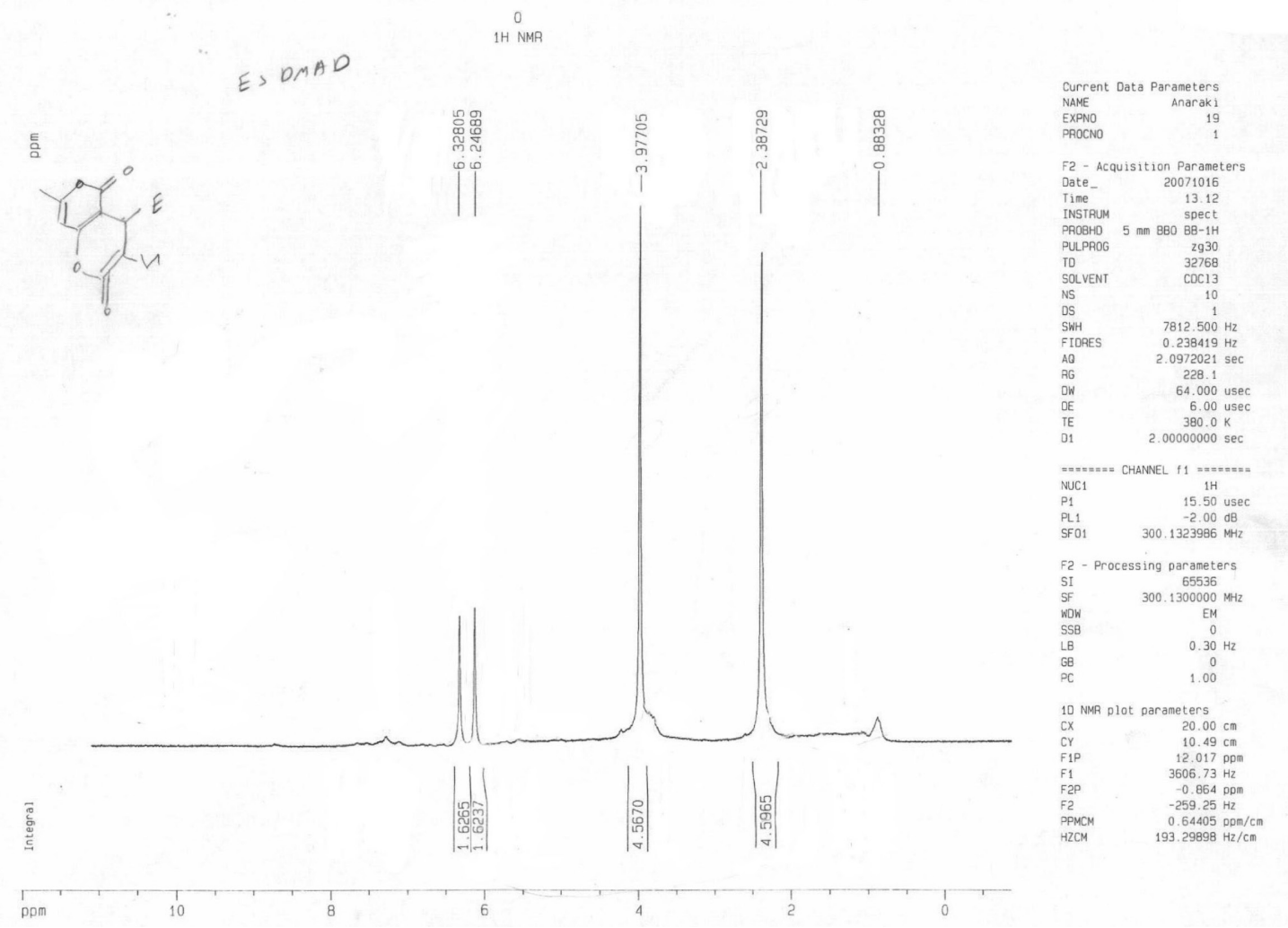

Figure S11. ${ }^{1} \mathrm{H}$ NMR spectrum of compound 15. 
D

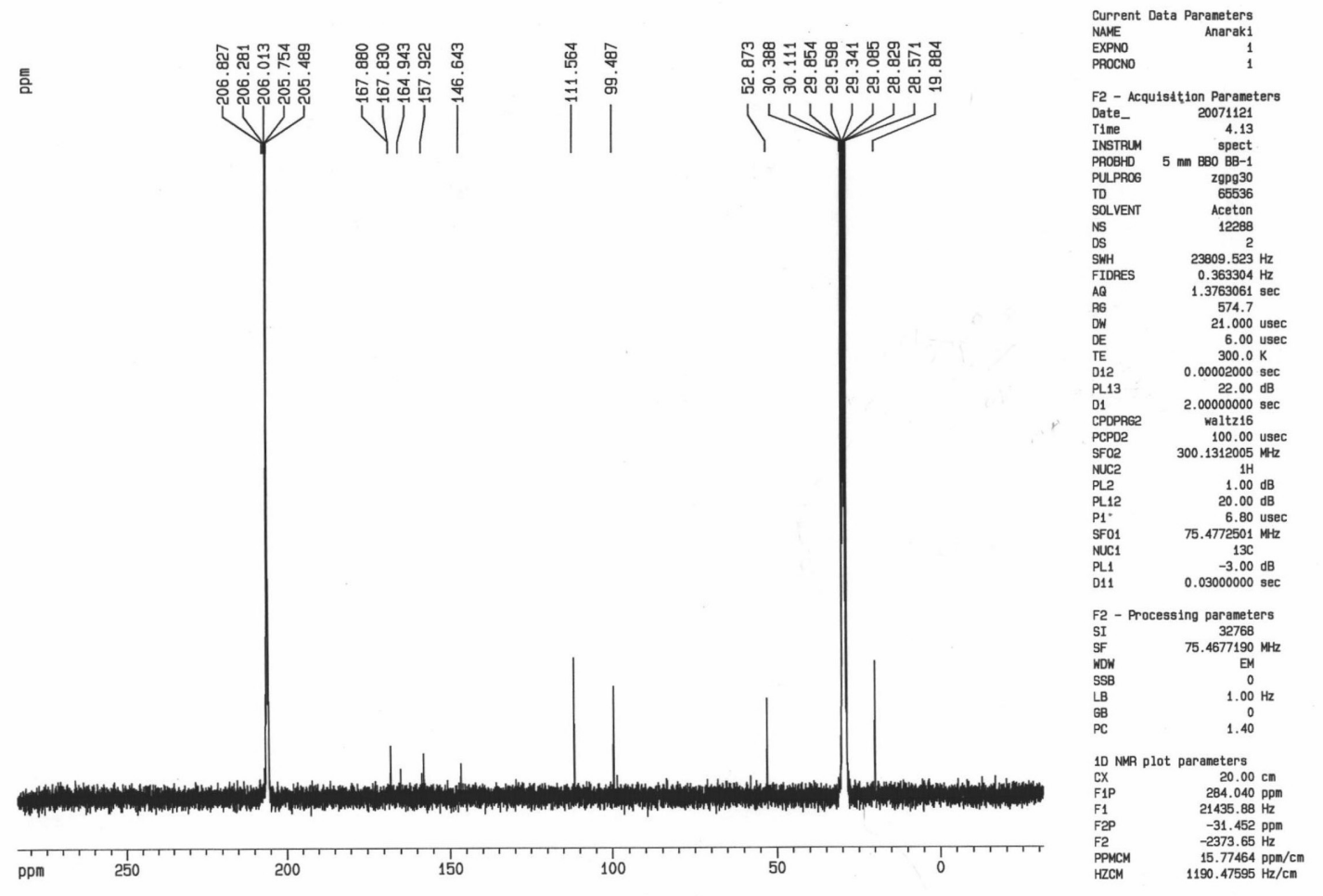

Figure S12. ${ }^{13} \mathrm{C}$ NMR spectrum of compound 15. 